\title{
Calculation of Bit Error Rates in Optical Systems with Silicon Photonic Wires
}

\author{
Jie You and Nicolae C. Panoiu
}

\begin{abstract}
A theoretical approach to calculate the bit error rate (BER) in optical systems containing silicon photonic wires (Si-PhWs) is presented. Specifically, the optical link consists of a single-mode silicon-on-insulator strip waveguide followed by a direct-detection optical receiver containing an optical filter, an ideal square-law photodetector, and an electrical filter. We assume that the optical input consists of a superposition of a nonreturnto-zero ON-OFF keying modulated optical signal and an additive white Gaussian noise, the BER of the transmitted optical signal being calculated by using the time domain Karhunen-Loève expansion method. The propagation of the optical signal in the $\mathrm{Si}-\mathrm{PhW}$ is described by employing both a rigorous theoretical model that incorporates all relevant linear and nonlinear optical effects and the mutual interaction between free-carriers and the optical field, as well as a linearized model valid in the low noise power regime. These analytical and computational tools are then used to comprehensively investigate the influence of the parameters characterizing the waveguide and optical signal on the transmission BER.
\end{abstract}

Index Terms-Silicon photonic wires, Optical interconnects, BER evaluation, direct-detection receiver, Karhunen-Loève expansion, nonlinear pulse propagation.

\section{INTRODUCTION}

$\mathbf{P}$ HOTONIC networks-on-chip (NoC) are becoming an integral part of high-performance computing (HPC) platforms, high-end data centers, and high-performance chip multiprocessors [1]-[3], a development underlined by the fact that rack-to-rack communications in some of the fastest HPC systems are nowadays performed optically [4]. It is envisioned that this trend of using optical communications at an eversmaller scale will continue to grow, so that in future HPC platforms optics will play the main role in node-to-node and even intra-node communications [3]. In this context, silicon photonics provides an ideal technological platform for the implementation of photonic NoC, both due to its potential for ultra-dense photonic integration, facilitated by the highindex contrast achievable in the silicon-on-insulator (SOI) platform, and its compatibility with the CMOS electronics platform [5]-[7]. In fact, all basic components of photonic NoC have already been implemented in the SOI platform, including optical amplifiers [8], [9], modulators [10]-[12], optical switches [13]-[15], receivers [16], [17], and frequency converters [18], [19].

Optical interconnects represent the backbone of future NoC, providing the key functionality of high-capacity point-to-point data communications. In order to become a viable alternative

Jie You was supported by a China Scholarship Council Studentship. The authors would like to thank P. Watts for many illuminating discussions.

Jie You and Nicolae C. Panoiu are with the Department of Electronic and Electrical Engineering, University College London, Torrington Place, London WC1E 7JE, United Kingdom to copper wires [20], on-chip optical interconnects must be able to facilitate ultrahigh bandwidth communication in a highly power-efficient manner and provide significant potential for scalability and on-chip device integration. For node-tonode or intra-node optical communications, silicon photonic wires (Si-PhWs) [21] implemented in the SOI platform present a compelling solution that satisfies all these requirements. The high-index contrast achievable in the SOI platform facilitates the control of optical power flow at the subwavelength scale whereas the large transparency window of silicon allows for ultrahigh bandwidth data communication [22]. In addition, due to their large optical nonlinearity, Si-PhWs are not only efficient chip-scale optical data streams conduits but can also be used as active devices that provide on-chip signal processing functionality, such as signal amplification, modulation, and frequency conversion.

In order to design highly efficient $\mathrm{NoC}$ and assess their performance, it is of critical importance to have a set of tools suitable for estimating the bit error rate (BER) of optical data streams transmitted among different nodes of the NoC. In particular, a reliable characterization of the performance of photonic NoC can be achieved via a bottom-up approach, in which one first determines at the physical layer the optical signal impairments introduced by each of the components of the NoC, this information being then used to evaluate at the system level the overall performance of the photonic network. To this end, a key step is to evaluate the BER introduced by $\mathrm{Si}-\mathrm{PhWs}$ and the influence of the waveguide and optical signal parameters on the transmission BER, by using theoretical models that fully capture the physics of the device both at optical and electrical levels.

In this paper, to the best of our knowledge for the first time, we perform a theoretical analysis of the transmission BER in a system consisting of a Si-PhW linked with a directdetection optical receiver containing an optical filter, an ideal square-law photodetector, and an electrical filter (see Fig. 1). We assume that the bandwidth of the optical filter is larger than the bit rate of the optical signal, whereas the bandwidth of the electrical filter is close to the bit rate. At the front-end of the system the optical field is assumed to be a superposition of an ON-OFF keying (OOK) modulated nonreturn-to-zero (NRZ) optical signal, with ON and OFF power values of $P_{0}$ and zero, respectively, and a stationary additive white Gaussian noise containing an in-phase and a quadrature component. For simplicity, we assume that these two noise components are uncorrelated, a constraint that can be easily relaxed if needed. To describe the optical field propagation in the Si-PhW we use a rigorous model [23] that incorporates linear and nonlinear optical effects, including free-carrier (FC) dispersion (FCD), 
FC absorption (FCA), self-phase modulation (SPM), and twophoton absorption (TPA), as well as the FCs dynamics and the interaction between the FCs and the optical field. A linearized system governing the optical noise dynamics in the presence of FCs is also derived and used to analyze the noise propagation in Si-PhWs. The system BER is calculated using the time domain Karhunen-Loève (KL) expansion method [24], an algorithm that has also been used to analyze the performance of optical fiber communication systems [25], [26].

The remaining of the paper is organized as follows. In Sec. II we present the theoretical model that describes the propagation of the optical signal in the Si-PhW, whereas in Sec. III we briefly outline the general formulation of the time domain KL expansion method used to evaluate the BER. The results of our analysis are presented in Sec. IV, the main conclusions of our study being summarized in the last section.

\section{Theoretical Model for Optical Signal Propagation In Silicon Photonic Wires}

We study the propagation of the optical signal in a Si-PhW by using a rigorous theoretical model introduced in [23] (for more details see also [27]-[29]), which describes the dynamics of the optical field and FCs as well as their mutual interaction. In mathematical terms, the coupled dynamics of the optical signal and FCs are governed by the following equations:

$$
\begin{aligned}
j \frac{\partial u}{\partial z} & -\frac{\beta_{2}}{2} \frac{\partial^{2} u}{\partial t^{2}}-\frac{j \beta_{3}}{6} \frac{\partial^{3} u}{\partial t^{3}}+\frac{j c \kappa}{2 n v_{g}}\left(\alpha_{i}+\alpha_{\mathrm{fc}}\right) u+\frac{\omega_{0} \kappa}{n v_{g}} \delta n_{\mathrm{fc}} u \\
& +\gamma\left(1+j \tau_{s} \frac{\partial}{\partial t}\right)|u|^{2} u=0, \\
\frac{\partial N}{\partial t} & =-\frac{N}{t_{c}}+\frac{3 \Gamma^{\prime \prime}}{4 \epsilon_{0} \hbar A^{2} v_{g}^{2}}|u|^{4},
\end{aligned}
$$

where $u(z, t)$ is the pulse envelope, measured in $\sqrt{\mathrm{W}}, z$ and $t$ are the distance along the $\mathrm{Si}-\mathrm{PhW}$ and time, respectively, $\beta_{n}=d^{n} \beta / d \omega^{n}$ is the $n$th order dispersion coefficient, $\omega_{0}$ is the carrier frequency, $\kappa$ measures the overlap between the optical mode and the (Si) active area of the waveguide, $v_{g}$ is the group-velocity, $\alpha_{i}$ is the intrinsic loss coefficient, which was set to $0.2 \mathrm{~dB} \mathrm{~cm}^{-1}$ in all our simulations unless otherwise specified, $t_{c}$ is the FC relaxation time (in our analysis we assumed $\left.t_{c}=0.5 \mathrm{~ns}\right), \delta n_{\mathrm{fc}}$ and $\alpha_{\mathrm{fc}}$ are the FC-induced refractive index change and $\mathrm{FC}$ loss coefficient, respectively, and are given by $\delta n_{\mathrm{fc}}=\sigma_{n} N$ and $\alpha_{\mathrm{fc}}=\sigma_{\alpha} N$, where $N$ is the FC density, $\sigma_{n}=-2.68 \times 10^{-26}(\lambda / \bar{\lambda})^{2}$ (in units of $\mathrm{m}^{3}$ ), and $\sigma_{\alpha}=1.45 \times 10^{-21}(\lambda / \bar{\lambda})^{2}$ (in units of $\mathrm{m}^{2}$ ) [28], the reference wavelength being $\bar{\lambda}=1550 \mathrm{~nm}$. The nonlinear properties of the waveguide are described by the nonlinear coefficient, $\gamma=3 \omega_{0} \Gamma / 4 \epsilon_{0} A v_{g}^{2}$, and the shock time scale, that is the characteristic response time of the optical nonlinearity, $\tau_{s}=\partial \ln \gamma / \partial \omega$, where $A$ and $\Gamma$ are the cross-sectional area and the effective third-order susceptibility of the waveguide, respectively. If the bit time window, $T_{0}$, is of the order of a few picoseconds or larger, as it is in our case, one can assume that the nonlinear response is instantaneous, namely $\tau_{s}=0$. Here and in what follows $\zeta^{\prime}\left(\zeta^{\prime \prime}\right)$ represents the real (imaginary) part of the complex number, $\zeta$.

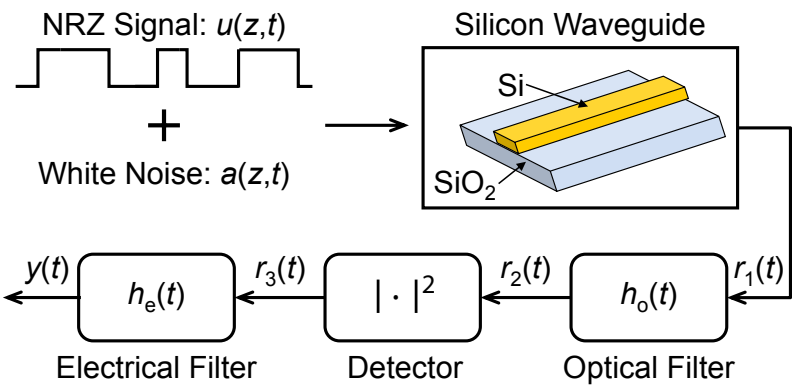

Fig. 1: Schematic of the investigated photonic system, consisting of a Si-PhW linked to a receiver containing an optical filter with impulse response function, $h_{o}(t)$, a photodetector, and an electrical filter with impulse response function, $h_{e}(t)$.

The terms in (1a) describe well known linear and nonlinear optical effects. Specifically, the second and third terms describe the second- and third-order dispersion, respectively, the fourth term corresponds to the intrinsic waveguide loss and FCA, the fifth term describes the FCD, whereas the last term represents nonlinear effects, namely the SPM, TPA, and frequency dispersion of the waveguide nonlinearity.

The superposition of the optical signal and noise propagating in the $\mathrm{Si}-\mathrm{PhW}$ can be expressed as,

$$
u(z, t)=[\sqrt{P(z)}+a(z, t)] e^{-j \Phi(z)},
$$

where $P(z)$ is the power of the CW signal, $a(z, t)$ is the complex additive Gaussian noise, and $\Phi(z)$ is a global phase shift. In the stationary regime, $\frac{\partial N}{\partial t}=0$, so that (1b) implies that the steady state FC density, $N_{s}$, is given by:

$$
N_{s}(z)=\frac{3 t_{c} \Gamma^{\prime \prime}}{4 \epsilon_{0} \hbar A^{2} v_{g}^{2}} P^{2}(z) \equiv \xi P^{2}(z) .
$$

In deriving this equation, we assumed that the power of the noise is much smaller than that of the optical signal. Note also that if one would include the bit statistics in this analysis then in (3) the parameter $\xi$ should be replaced by $\xi / 2$, because for a data stream that on average contains the same number of "1"- and "0"-bits the averaged square power, $\left\langle P^{2}\right\rangle=P^{2} / 2$.

In order to linearize (1) w.r.t. the noise amplitude, $a(z, t)$, we substitute (2) into (1a) and discard all quadratic and higherorder terms in $a(z, t)$. Then, in conjunction with (3), the zeroth- and first-order of (1a) become:

$$
\begin{aligned}
\frac{d P}{d z}= & -\frac{c \kappa}{n v_{g}} \alpha_{i} P-\frac{c \kappa}{n v_{g}} \sigma_{\alpha} \xi P^{3}-2 \gamma^{\prime \prime} P^{2} \\
\frac{d \Phi}{d z}= & -\frac{\omega_{0} \kappa}{n v_{g}} \sigma_{n} \xi P^{2}-\gamma^{\prime} P \\
\frac{\partial a}{\partial z}= & -j \frac{\beta_{2}}{2} \frac{\partial^{2} a}{\partial t^{2}}+\frac{\beta_{3}}{6} \frac{\partial^{3} a}{\partial t^{3}}-\frac{c \kappa}{2 n v_{g}} \sigma_{\alpha} \xi P^{2}\left(3 a+2 a^{*}\right) \\
& +j \frac{2 \omega_{0} \kappa}{n v_{g}} \sigma_{n} \xi P^{2}\left(a+a^{*}\right)-\frac{c \kappa}{2 n v_{g}} \alpha_{i} a \\
& +j \gamma P\left(a+a^{*}\right)-\gamma^{\prime \prime} P a,
\end{aligned}
$$

where the symbol "*” means complex conjugation. These equations show that the power can be calculated independently on the phase and noise amplitude, its decay being due to 
intrinsic losses, FCA, and TPA. Note also that the total phase variation of the optical field has two sources, namely the FCD and nonlinearly induced phase shifts.

By adding to and subtracting (4c) from its complex conjugate, two coupled differential equations are obtained for the inphase and quadrature noise components, $a^{\prime}(z, t)$ and $a^{\prime \prime}(z, t)$, respectively. Taking the Fourier transform of both sides of the resulting equations leads to the following system of coupled ordinary differential equations:

$$
\begin{aligned}
\frac{d A^{\prime}}{d z}= & -\frac{\beta_{2}}{2} \Omega^{2} A^{\prime \prime}-j \frac{\beta_{3}}{6} \Omega^{3} A^{\prime}-\frac{c \kappa}{2 n v_{g}}\left(\alpha_{i}+5 \sigma_{\alpha} \xi P^{2}\right) A^{\prime} \\
& -3 \gamma^{\prime \prime} P A^{\prime}, \\
\frac{d A^{\prime \prime}}{d z}= & \frac{\beta_{2}}{2} \Omega^{2} A^{\prime}-j \frac{\beta_{3}}{6} \Omega^{3} A^{\prime \prime}-\frac{c \kappa}{2 n v_{g}}\left(\alpha_{i}+\sigma_{\alpha} \xi P^{2}\right) A^{\prime \prime} \\
& +\frac{4 \omega_{0} \kappa}{n v_{g}} \sigma_{n} \xi P^{2} A^{\prime}+2 \gamma^{\prime} P A^{\prime}-\gamma^{\prime \prime} P A^{\prime \prime}
\end{aligned}
$$

where $\Omega=\omega-\omega_{0}$ and $A^{\prime}(z, \Omega)=\mathcal{F}\left\{a^{\prime}(z, t)\right\}$ and $A^{\prime \prime}(z, \Omega)=\mathcal{F}\left\{a^{\prime \prime}(z, t)\right\}$ are the Fourier transforms of the in-phase and quadrature noise components, respectively.

The full model (1) and its linearized version (5) can be extended to other devices, too, the main difference being that the resulting mathematical description could potentially become much more intricate. For example, waveguide splitters, ring modulators coupled to a waveguide, multi-wavelength signals propagating in single- or multi-mode waveguides can all be described by systems of coupled equations similar to (1) and its linearized version (5). Therefore, the approach presented in this study can be applied to a multitude of chip-level photonic devices, thus underlying the generality of our approach.

We have determined the optical field at the output of the Si$\mathrm{PhW}$ both by integrating the full system (1), using a standard split-step Fourier method, and also by solving the linearized system (4a), (4b), and (5) via a 5th order Runge-Kutta method. In the latter case, we first found the spectra of the noise components, then by inverse Fourier transforming these spectra we calculated the optical noise in the time domain. Moreover, in order to gain a more complete understanding of the factors that affect the BER, we considered Si-PhWs with both normal and anomalous dispersion. Thus, since the linear and nonlinear properties of stripe $\mathrm{Si}-\mathrm{PhW}$ depend strongly on the waveguide geometry [23], the waveguide parameters (dispersion and nonlinear coefficients) can be varied over a wide range of values by properly choosing the waveguide height, $h$, and its width, $w$. In particular, unless otherwise specified, we assumed that the Si-PhW has constant height and width, its optical waveguide parameters being thus independent on the distance along the waveguide, $z$. Specifically, the waveguide with normal dispersion (called waveguide $A$ ) is characterized by $\beta_{2}=0.26 \mathrm{ps}^{2} \mathrm{~m}^{-1}, \beta_{3}=2.8 \times 10^{-3} \mathrm{ps}^{3} \mathrm{~m}^{-1}$, $\gamma^{\prime}=183.1 \mathrm{~W}^{-1} \mathrm{~m}^{-1}$, and $\gamma^{\prime \prime}=55.8 \mathrm{~W}^{-1} \mathrm{~m}^{-1}$, whereas the waveguide with anomalous dispersion (called waveguide B) has $\beta_{2}=-0.2 \mathrm{ps}^{2} \mathrm{~m}^{-1}, \beta_{3}=3.8 \times 10^{-3} \mathrm{ps}^{3} \mathrm{~m}^{-1}$, $\gamma^{\prime}=207.7 \mathrm{~W}^{-1} \mathrm{~m}^{-1}$, and $\gamma^{\prime \prime}=63.3 \mathrm{~W}^{-1} \mathrm{~m}^{-1}$. These values correspond to a Si-PhW with width $w=800 \mathrm{~nm}(w=675 \mathrm{~nm})$ for the normal (anomalous) dispersion case, whereas in both cases the waveguide height, $h=250 \mathrm{~nm}$ [30].

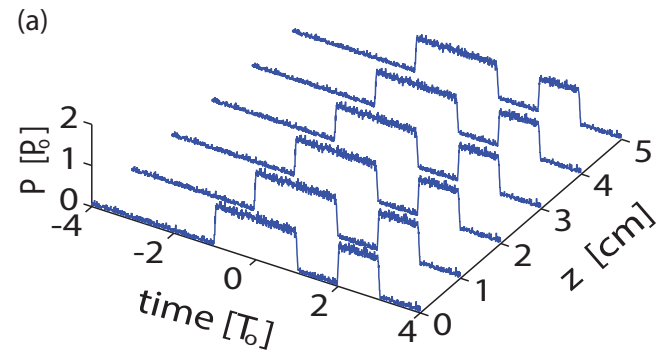

(b)
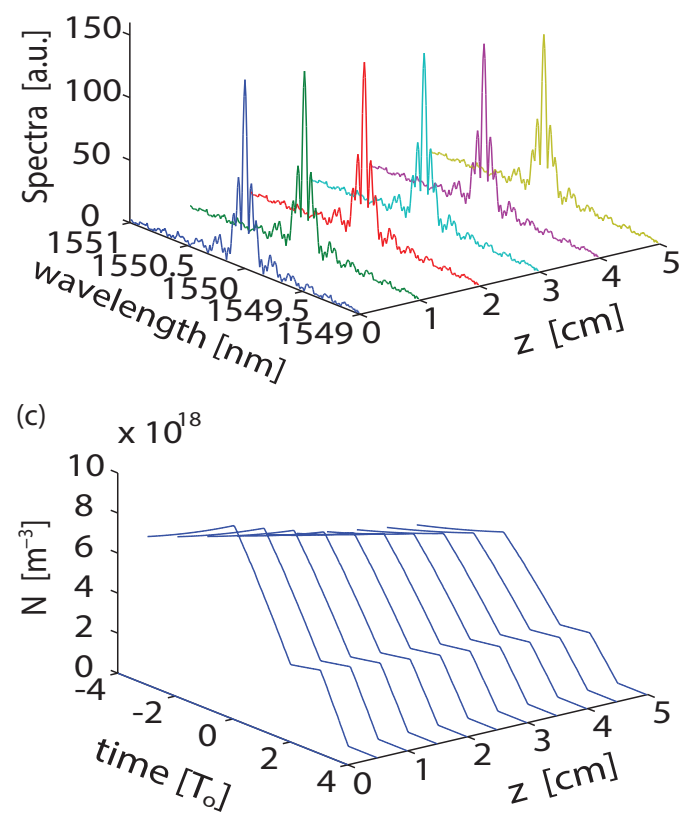

Fig. 2: (a) Time and (b) spectral domain evolution of a noisy signal with $P_{0}=5 \mathrm{~mW}$ and $T_{0}=100 \mathrm{ps}$ in a $5 \mathrm{~cm}$-long Si$\mathrm{PhW}$ with anomalous dispersion (see the text for the values of $\beta_{2}, \beta_{3}$, and $\gamma$ ). (c) Carrier density variation along the waveguide.

An example of time and wavelength domain evolution of a noisy signal in a $5 \mathrm{~cm}$-long $\mathrm{Si}-\mathrm{PhW}$ with anomalous dispersion is shown in Fig. 2, the bit sequence being "01011000". For completeness, we also show in Fig. 2(c) the dynamics of the photogenerated FCs. It can be seen that the optical field is fairly weakly distorted during propagation, which means that for the optical power considered in these simulations the nonlinear effects are small. The most notable feature revealed by these plots is the signal decay, which is due to the intrinsic losses, FCA, and TPA. The generation of the FCs that produce FCA is illustrated in Fig. 2(c), where the increase in the FC density induced by each "1"-bit can be clearly seen.

In order to determine the accuracy with which the linearized system (4) describes the propagation of the optical field in the Si-PhW, we calculated the signal and noise at the backend of the waveguide by using both the linearized model and full system (1). The conclusions of this analysis, summarized in Fig. 3, suggest that the linearized system describes fairly accurately the dynamics of the $\mathrm{CW}$ signal and noise, especially when the noise power is small. Thus, Figs. 3(b) and 3(c) show that both models predict a larger parametric amplification of 

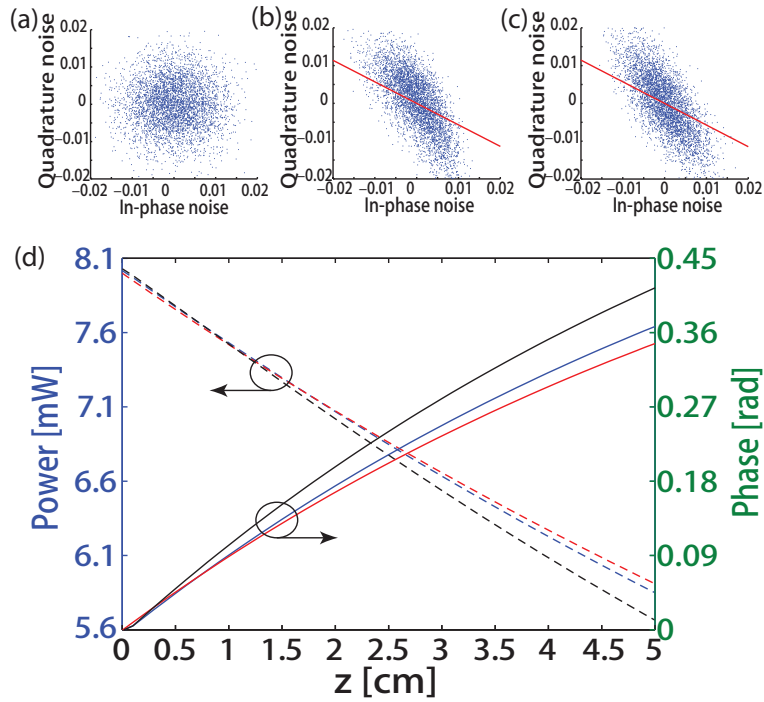

Fig. 3: (a) In-phase and quadrature noise components at the input of the Si-PhW and (b), (c) waveguide output, determined from the full system (1) and linearized system (4), respectively. The propagation length, $L=5 \mathrm{~cm}$, and $\mathrm{SNR}=20 \mathrm{~dB}$. The red lines indicate the average phase of the noise. The $\mathrm{Si}-\mathrm{PhW}$ is the same as in Fig. 2. (d) Power $P(z)$ and phase $\Phi(z)$ calculated using the linearized system (red lines) and full system for $\mathrm{SNR}=20 \mathrm{~dB}$ (blue lines) and $\mathrm{SNR}=15 \mathrm{~dB}$ (black lines).

the quadrature noise (a finding also supported by the power spectral densities of the two noises, not shown here) and similar values of the average phase, $\vartheta$, of the noise, which is equal to the slope of the red lines in these plots. This is a known effect, a larger parametric gain amplification of the quadrature noise being observed in optical fiber systems, too [31]. In the case when the linearized system was used, the phase $\vartheta$ was calculated from the relation, $\vartheta=\mathbb{E}\{\arg [a(z, t)]\}$, where $\mathbb{E}\{\cdot\}$ denotes the statistical expectation operator. When the full system was used, the $\mathrm{CW}$ signal parameters were extracted from the relation, $\sqrt{P(z)} e^{-j \Phi(z)}=\mathbb{E}\{u(z, t)\}$, and then the noise was found as $a(z, t)=[u(z, t)-\mathbb{E}\{u(z, t)\}] e^{j \Phi(z)}$.

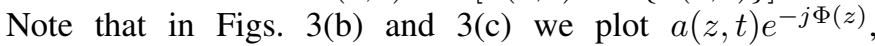
calculated at $z=L=5 \mathrm{~cm}$.

A good agreement between the two models can also be observed in their predictions of the dependence of the CW signal power and phase on the distance, $z$, as per Fig. 3(d). Expectedly, the differences between the results inferred from the two models decrease with the signal-to-noise ratio (SNR) as the effects due to the nonlinear noise propagation and noise interaction with FCs, which are neglected in the linearized model, become less important as the SNR increases. In particular, as compared to the full system, the linearized system overestimates the power of the $\mathrm{CW}$ signal and underestimates its phase. In our simulations we define the SNR of the optical signal at the front-end of the $\mathrm{Si}-\mathrm{PhW}$ as the ratio between the power of the CW signal, $P_{0}$, and the average of the sum of the powers of the in-phase and quadrature noise components,

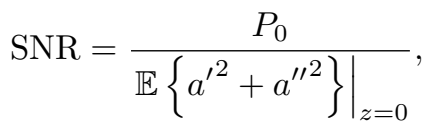

\section{Calculation of BER}

In this section, we briefly outline the analytical method used to calculate the transmission BER at the back-end of the receiver, namely the time-domain KL series expansion method [25], [26]. We assume that the direct-detection receiver is composed of a Lorentzian optical filter with impulse response, $h_{o}(t)$, followed by an ideal photodetector, and an integrateand-dump electrical filter, whose impulse response is $h_{e}(t)$. The electrical noise of the receiver has not been taken into account, as in most cases it can be neglected.

We represent the complex envelope of the optical signal at the back-end of the Si-PhW as $r_{1}(t)=S+a_{i}(t)+j a_{q}(t)$, where $a_{i}(t)$ and $a_{q}(t)$ are the in-phase and quadrature noise components, respectively, and $S$ is the amplitude of the $\mathrm{CW}$ signal. We consider that $S=0\left(S^{2}=P\right)$ when a "0" ("1") is transmitted, where $P$ is the signal power at the waveguide output. Moreover, it is assumed that the carrier propagates unchanged through the optical filter, which amounts to $H_{o}(0)=1$ with $H_{o}(f)=\mathcal{F}\left\{h_{o}(t)\right\}$. Hence, the signal after the optical filter can be written as, $r_{2}(t)=S+v_{i}(t)+j v_{q}(t)$, where $v_{i, q}(t)=h_{o}(t) \otimes a_{i, q}(t)$. After passing through the ideal square-law photodetector and the electrical filter, the electrical signal, $y(t)=h_{e}(t) \otimes\left|r_{2}(t)\right|^{2}$, so that at the back-end of the receiver it is given by the following expression:

$$
y(t)=\int_{-\infty}^{\infty} h_{e}(t)\left\{\left[S+v_{i}\left(t-t^{\prime}\right)\right]^{2}+v_{q}^{2}\left(t-t^{\prime}\right)\right\} d t^{\prime} .
$$

The noise components at the output of the $\mathrm{Si}-\mathrm{PhW}$ are assumed to be stationary and completely determined by their power spectral density matrix [24]:

$$
\underline{\underline{\mathcal{G}}}_{a}(f)=\left(\begin{array}{cc}
\mathcal{G}_{a}^{i}(f) & \mathcal{G}_{a}^{i q}(f) \\
\mathcal{G}_{a}^{q i}(f) & \mathcal{G}_{a}^{q}(f)
\end{array}\right),
$$

where $\mathcal{G}_{a}^{i}$ and $\mathcal{G}_{a}^{q}$ are the power spectral densities of the inphase and quadrature noise components and $\mathcal{G}_{a}^{i q}=\mathcal{G}_{a}^{q i}$ is their cross-spectral density. Note that even if at the input of the Si-PhW the in-phase and quadrature noise components are uncorrelated, the mutual interaction mediated by FCs and optical nonlinearity makes them to become correlated by the time they reach the back-end of the waveguide.

Upon passing through the optical filter, the power spectral density matrix of the noise, $\underline{\underline{\mathcal{G}}}_{v}(f)$, becomes [24]:

$$
\underline{\underline{\mathcal{G}}}_{v}(f)=\underline{\underline{\mathcal{H}}}_{o}(f) \cdot \underline{\underline{\mathcal{G}}}_{a}(f) \cdot \underline{\underline{\mathcal{H}}}_{o}^{\dagger}(f),
$$

where " $\dagger$ " represents Hermitian conjugation operation and

$$
\underline{\underline{\mathcal{H}}}_{o}=\left(\begin{array}{cc}
H_{o}^{i}(f) & -H_{o}^{q}(f) \\
H_{o}^{q}(f) & H_{o}^{i}(f)
\end{array}\right) .
$$

Here, $H_{o}^{i}(f)=\mathcal{F}\left\{h_{o}^{\prime}(t)\right\}$ and $H_{o}^{q}(f)=\mathcal{F}\left\{h_{o}^{\prime \prime}(t)\right\}$ are the Fourier transform of the real and imaginary parts of the optical filter impulse response, respectively.

To calculate the BER, the signal, $S$, and both components of the noise, $v_{i}(t)$ and $v_{q}(t)$, are expanded in KL series [32], the expansion coefficients being random variables whose expectation values and correlation matrix can be calculated following an algorithm described in [25], [26]. After one diagonalizes this correlation matrix, the moment-generating 
function (MGF), $\Psi_{y}(\zeta)$, of the random variable $y$ defined by (7) can be written as [33]:

$$
\Psi_{y}(\zeta)=\mathbb{E}\left\{e^{-\zeta y}\right\}=\prod_{\alpha=1}^{M} \frac{\exp \left(-\frac{\left|\eta_{\alpha}\right|^{2} \zeta}{1+2 \delta_{\alpha} \zeta}\right)}{\sqrt{1+2 \delta_{\alpha} \zeta}},
$$

where $\delta_{\alpha}$ are the eigenvalues of the correlation matrix, whose dimension is $M \times M$, and $\eta_{\alpha}$ are the expectation values of $y$, determined in the basis in which the correlation matrix is diagonal (for a detailed description of this algorithm see [25]).

Finally, the transmission BER of the system can be evaluated from the following relation:

$$
\mathcal{P}=\frac{1}{2}\left[\mathcal{P}\left(y>y_{t h} \mid S=0\right)+\mathcal{P}\left(y<y_{t h} \mid S=\sqrt{P}\right)\right],
$$

where the first (second) term is the probability for an error to occur when a "0" (" 1 ") bit is transmitted and $y_{t h}$ is the decision threshold. Using the Riemann-Fourier inversion formula of the MGF, these probabilities can be found as [34]:

$$
\begin{aligned}
& \mathcal{P}\left(y>y_{t h} \mid S=0\right)=-\int_{-\left|\zeta_{0}\right|-\infty}^{-\left|\zeta_{0}\right|+\infty} \frac{\Psi_{y}(\zeta \mid S=0)}{2 \pi j \zeta} e^{\zeta y_{t h}} d \zeta, \\
& \mathcal{P}\left(y<y_{t h} \mid S=\sqrt{P}\right)=\int_{\left|\zeta_{0}\right|-\infty}^{\left|\zeta_{0}\right|+\infty} \frac{\Psi_{y}(\zeta \mid S=\sqrt{P})}{2 \pi j \zeta} e^{\zeta y_{t h}} d \zeta,
\end{aligned}
$$

where $\zeta_{0}$ is a real constant that defines the integration path in the complex plane, $\zeta$. As a final note on the BER calculation, we stress that this KL-based method produces significantly more accurate results when the parametric gain amplification of the noise cannot be neglected, as compared to the commonly used Gaussian approximation [25].

\section{Results AND Discussion}

In order to illustrate how our approach can be applied in practical cases to calculate the system BER, we consider

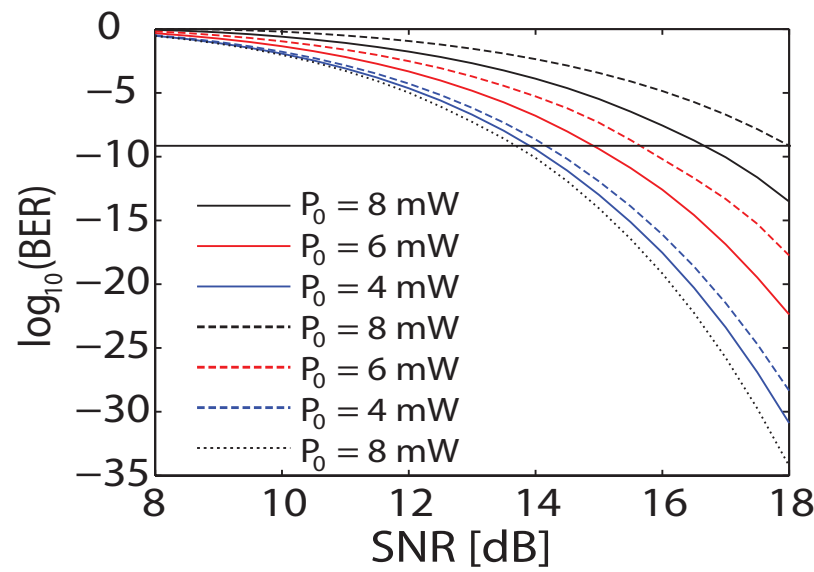

Fig. 4: System BER vs. SNR, calculated for Si-PhWs with normal (solid line) and anomalous (dashed line) dispersion (see the text for the values of $\beta_{2}, \beta_{3}$, and $\gamma$ ). The waveguide length, $L=5 \mathrm{~cm}$. The dotted line indicates the BER in the case of a system without the silicon waveguide. The horizontal black solid line corresponds to a BER of $10^{-9}$.
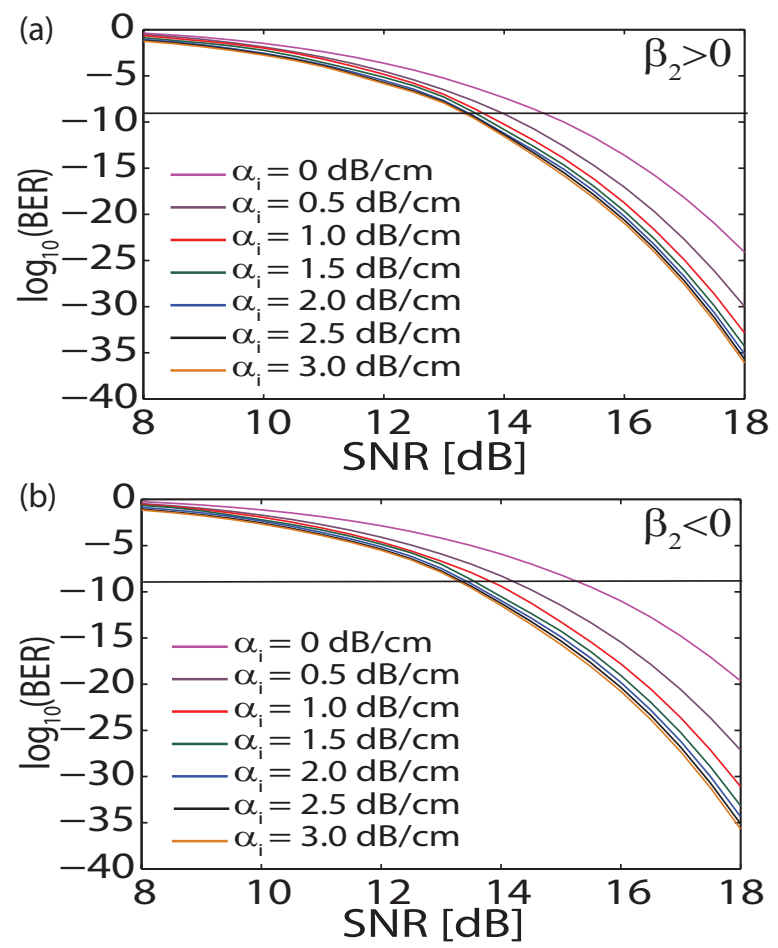

Fig. 5: System BER vs. SNR, calculated for several different values of the waveguide loss coefficient, $\alpha_{i}$. The panels (a) and (b) correspond to waveguides $A\left(\beta_{2}>0\right)$ and $B\left(\beta_{2}<0\right)$, respectively. In all simulations $P_{0}=5 \mathrm{~mW}$ and $L=5 \mathrm{~cm}$. The horizontal black solid line corresponds to a BER of $10^{-9}$.

a single-channel OOK system $\left(\lambda_{0}=1550 \mathrm{~nm}\right)$ with NRZ pulses in a back-to-back configuration, the bit window being $T_{0}=100 \mathrm{ps}$ throughout our investigations. To model the direct-detection receiver, we assume that the electrical filter is a low-pass integrate-and-dump filter with the $3-\mathrm{dB}$ bandwidth equal to $B_{e}=10 \mathrm{~Gb} \mathrm{~s}^{-1}$, whereas the optical filter is a bandpass Lorentzian with 3-dB bandwidth, $B_{o}=4 B_{e}$. Specifically, the two filters are described by the following transfer functions,

$$
\begin{aligned}
& H_{o}^{i}(f)=\frac{\Gamma_{o}^{2}}{f^{2}+\Gamma_{o}^{2}}, \quad H_{o}^{q}(f)=-\frac{\Gamma_{o} f}{f^{2}+\Gamma_{o}^{2}}, \\
& H_{e}^{i}(f)=\left\{\begin{array}{cc}
1, & |f| \leq B_{e} / 2 \\
0, & |f|>B_{e} / 2
\end{array} \quad H_{e}^{q}(f)=0,\right.
\end{aligned}
$$

where $\Gamma_{o}=B_{o} / 2$.

In our calculations of the system BER, we considered $\mathrm{Si}$ $\mathrm{PhWs}$ with both normal and anomalous dispersion and in both cases we assumed that the waveguide length, $L=5 \mathrm{~cm}$. For comparison, we also examined the case of a system without the $\mathrm{Si}-\mathrm{PhW}$, so that the contribution of the waveguide to the system BER can be easily assessed. The dependence of the system BER on the SNR, calculated for several values of the input power, $P_{0}$, is presented in Fig. 4 . The results summarized in this figure demonstrate that a better system performance is achieved in the normal dispersion regime, which is primarily due to the fact that the Si-PhW with anomalous dispersion has a larger nonlinear coefficient and consequently it generates a larger parametric gain amplification of the noise. Moreover, 


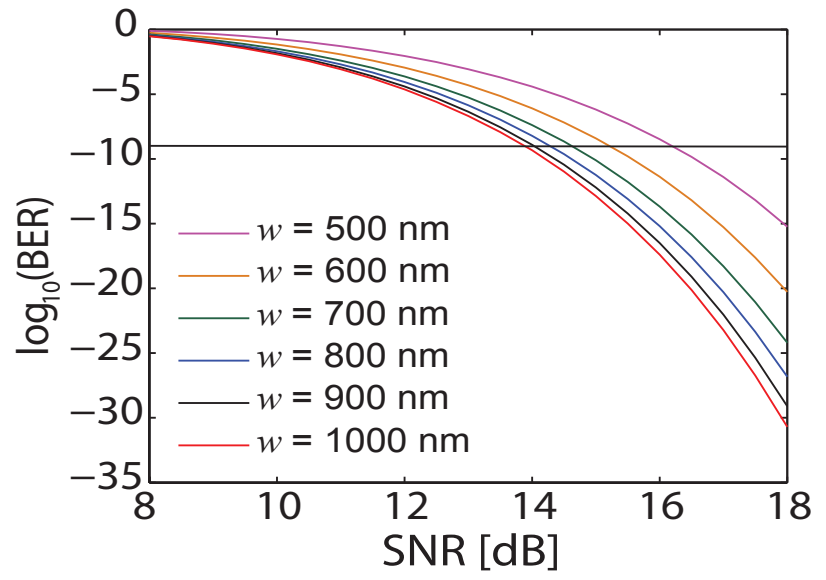

Fig. 6: System BER vs. SNR, calculated for Si-PhWs with different width, $w$. The waveguide parameters for all widths are given in Table I. In all cases $P_{0}=5 \mathrm{~mW}$ and $L=5 \mathrm{~cm}$. The horizontal black solid line indicates a BER of $10^{-9}$.

because the parametric gain also increases with the optical power, one expects that increasing $P_{0}$ would lead to larger BER, a conclusion fully validated by the plots in Fig. 4. This figure also shows that the $\mathrm{Si}-\mathrm{PhW}$ has a significant contribution to the signal degradation, especially for large SNR.

Depending on the width of the waveguide and specific fabrication processes, the intrinsic loss coefficient, $\alpha_{i}$, can usually vary from $0.03 \mathrm{~dB} \mathrm{~cm}^{-1}$ to more than $3 \mathrm{~dB} \mathrm{~cm}^{-1}$. We therefore considered the two Si-PhWs with positive and negative dispersion coefficient and in both cases calculated the system BER for several values of $\alpha_{i}$. In all these calculations we chose $P_{0}=5 \mathrm{~mW}$ and $L=5 \mathrm{~cm}$. The results of these simulations, plotted in Fig. 5, demonstrate that as the waveguide loss coefficient increases the system performance improves, which is reflected in a smaller transmission BER. This conclusion is in agreement with the dependence of BER on pulse power illustrated in Fig. 4. To be more specific, when $\alpha_{i}$ increases the power of the signal upon its propagation in the $\mathrm{Si}-\mathrm{PhW}$ decreases and therefore a smaller parametric gain amplification of the noise is produced. This results in a larger SNR at the output facet of the waveguide and consequently a reduced BER. Note also that, similarly to the dependence illustrated in Fig. 4, the variation of the BER with $\alpha_{i}$, for the same value of the SNR, is smaller for the waveguide $A$ $\left(\beta_{2}>0\right)$ as compared to the case of waveguide $B\left(\beta_{2}<0\right)$.

Due to the strong confinement of light in Si-PhWs with submicrometer transverse size, the waveguide parameters characterizing their linear and nonlinear optical properties are strongly dependent on the waveguide width. It is therefore of particular interest to investigate the dependence on the waveguide width of the system BER. To this end, we considered several Si-PhWs with width ranging from $500 \mathrm{~nm}$ to $1000 \mathrm{~nm}$ and constant height, $h=250 \mathrm{~nm}$, and for all these waveguides we determined their waveguide parameters; the corresponding values are presented in Table I. We stress that for the range of widths considered here the waveguides are single-mode [30]. Note that as the waveguide width varies within the specified bounds, the second-order dispersion coefficient, $\beta_{2}$, changes from anomalous to normal dispersion regime.

After the waveguide parameters have been determined, we have calculated the system BER corresponding to each of the waveguides considered. In all cases we set $P_{0}=5 \mathrm{~mW}$ and $L=5 \mathrm{~cm}$. As illustrated in Fig. 6, the main conclusion that can be drawn from this analysis is that the BER decreases as the waveguide width increases. This result can be readily understood if one considers the variation of the waveguide nonlinear coefficient, $\gamma^{\prime}$, with the waveguide width, $w$ (see Table I). Thus, it can be seen that as $w$ increases the waveguide nonlinearity decreases, and therefore the parametric gain amplification is weaker. As a result, the SNR increases, which leads to a smaller BER.

Since both the nonlinear optical effects and FC dynamics are mainly determined by the optical power, we proceeded to analyze in more in-depth the dependence of the system BER on the input power of the $\mathrm{CW}$ signal. The results of this study, determined for the waveguides $A$ and $B$ described in Sec. II, are presented in Fig. 7 as contour maps of $\log _{10}$ (BER). While confirming the conclusions illustrated in Fig. 4, it can be seen that the maps in Fig. 7 reveal additional features. Thus, at low power the BER is almost independent on $P_{0}$, which is explained by the fact that in this situation the signal and noise propagates in the linear regime. If the power increases beyond $P_{0} \approx 5 \mathrm{~mW}$, however, the FCs generated via TPA as well as the nonlinear effects begin to strongly affect the signal propagation and as a result the BER varies nonlinearly
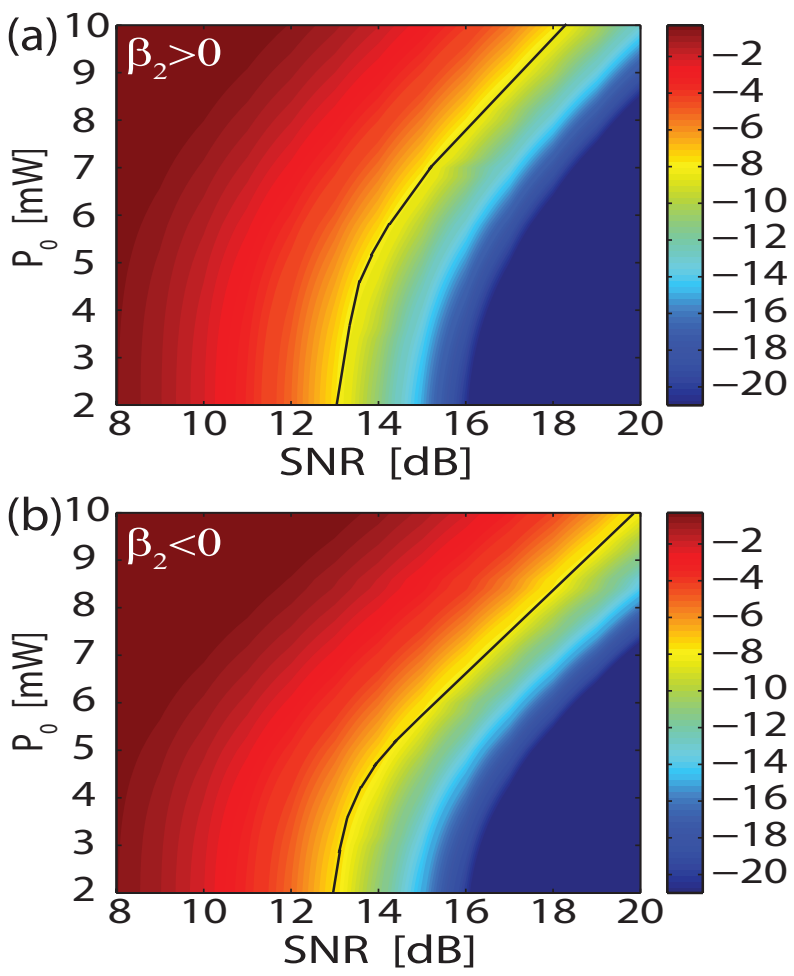

Fig. 7: Contour maps of $\log _{10}(\mathrm{BER})$ vs. power and SNR. (a), (b) correspond to $\mathrm{Si}-\mathrm{PhWs}$ with normal and anomalous dispersion, respectively, the waveguides being the same as in Fig. 4. The black contours correspond to $\mathrm{BER}=10^{-9}$. 
TABLE I: Waveguide parameters used to obtain the results presented in Fig. 6

\begin{tabular}{c|c|c|c|c|c|c}
\hline \hline$w[\mathrm{~nm}]$ & $c / v_{g}$ & $\beta_{2}\left[\mathrm{ps}^{2} \mathrm{~m}^{-1}\right]$ & $\beta_{3}\left[\mathrm{ps}^{3} \mathrm{~m}^{-1}\right]$ & $\kappa$ & $\gamma^{\prime}\left[\mathrm{W}^{-1} \mathrm{~m}^{-1}\right]$ & $\gamma^{\prime \prime}\left[\mathrm{W}^{-1} \mathrm{~m}^{-1}\right]$ \\
\hline \hline 500 & 4.2 & -1.2455 & $3.73 \times 10^{-3}$ & 0.9399 & 251.64 & 76.68 \\
\hline 600 & 4.05 & -0.595 & $4.33 \times 10^{-3}$ & 0.9552 & 225.16 & 68.60 \\
\hline 700 & 3.96 & -0.0921 & $3.57 \times 10^{-3}$ & 0.9624 & 202.29 & 61.63 \\
\hline 800 & 3.93 & 0.258 & $2.80 \times 10^{-3}$ & 0.9663 & 183.08 & 55.77 \\
\hline 900 & 3.87 & 0.504 & $2.21 \times 10^{-3}$ & 0.9685 & 166.84 & 50.83 \\
\hline 1000 & 3.84 & 0.684 & $1.73 \times 10^{-3}$ & 0.9699 & 153.02 & 46.62 \\
\hline \hline
\end{tabular}
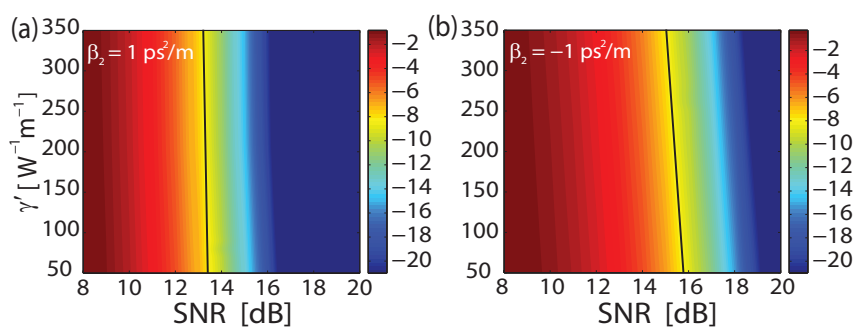

Fig. 8: Maps of $\log _{10}(\mathrm{BER})$ vs. $\gamma^{\prime}$ and SNR. (a), (b) correspond to $\mathrm{Si}-\mathrm{PhWs}$ with normal and anomalous dispersion, respectively. In both cases $\gamma^{\prime \prime} / \gamma^{\prime}=0.3, P_{0}=5 \mathrm{~mW}$, and $L=5 \mathrm{~cm}$. The black contours indicate a BER of $10^{-9}$.

with $P_{0}$. Moreover, as expected, low signal degradation is observed at small $P_{0}$ and large SNR (the boundary of the domain where the BER has values that are tolerable in regular practical systems, namely $\log _{10}(\mathrm{BER}) \leq-9$, is shown as the black contour in Fig. 7).

Additional insights into the contribution of nonlinear effects to the system signal degradation are provided by the dependence of the system BER on the waveguide nonlinear coefficient, the corresponding contour maps being presented in Fig. 8. We have investigated $\mathrm{Si}-\mathrm{PhWs}$ with normal $\left(\beta_{2}=1 \mathrm{ps}^{2} \mathrm{~m}^{-1}\right)$ and anomalous $\left(\beta_{2}=-1 \mathrm{ps}^{2} \mathrm{~m}^{-1}\right)$ dispersion, in both cases the ratio $\gamma^{\prime \prime} / \gamma^{\prime}=0.3$ being kept constant. A comparison between the results shown in Fig. 8(a) and Fig. 8(b) reveals several interesting features of the system BER. Thus, for Si-PhWs with normal dispersion the BER depends only slightly on $\gamma^{\prime}$, as in this case the parametric gain is relatively small. By contrast, the BER in the anomalous dispersion regime depends much stronger on $\gamma^{\prime}$, due to a much larger parametric gain amplification of the noise. In particular, for the same waveguide $(\gamma)$ and optical signal ( $P_{0}$ and SNR) parameters, the system signal degradation is more pronounced in the anomalous dispersion regime.

A key property one employs when assessing the feasibility of using $\mathrm{Si}-\mathrm{PhWs}$ as on-chip optical interconnects is the relationship between the waveguide transmission BER and the waveguide length, $L$. In order to characterize this dependence, we have determined the system BER as a function of $L$, the main results of this study being summarized in Fig. 9. We considered Si-PhWs with normal and anomalous dispersion, the calculations being performed for a $\mathrm{CW}$ signal with power, $P_{0}=5 \mathrm{~mW}$. One important result illustrated by this figure is that a BER smaller than $10^{-9}$ can be achieved even when the waveguide length is as large as $10 \mathrm{~cm}$, provided that the SNR

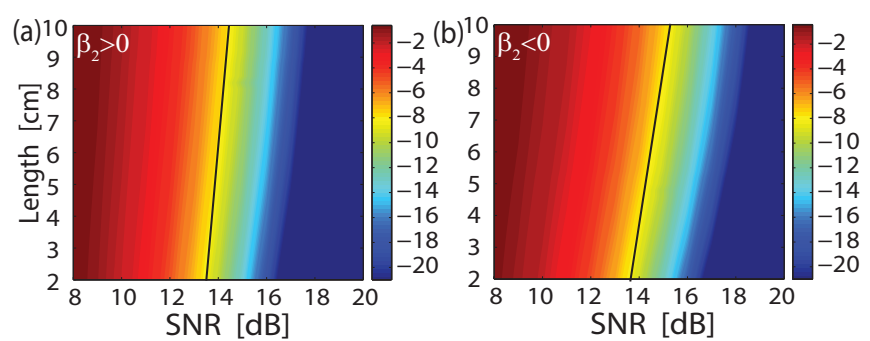

Fig. 9: Contour maps of $\log _{10}(\mathrm{BER})$ vs. waveguide length and SNR. Panels (a) and (b) correspond to Si-PhWs with normal and anomalous dispersion, respectively, the waveguides being the same as in Fig. 4. The input power is $P_{0}=5 \mathrm{~mW}$. The black contours correspond to a BER of $10^{-9}$.

is suitably large, namely $\mathrm{SNR} \gtrsim 15 \mathrm{~dB}$. Moreover, as before, it can be seen that the system signal degradation is larger in the case of waveguides with anomalous dispersion.

\section{CONCLUSION}

In conclusion, we have introduced a novel approach to the evaluation of bit error rates in optical systems containing silicon photonic wires. In order to describe the evolution of the mutually interacting optical field and free-carriers in the silicon photonic wire we employed both a rigorous theoretical model that incorporates all the linear and nonlinear physical effects and the linearized version of this full model, valid in the low noise power limit. The signal degradation in a link containing such a waveguide and a direct-detection optical receiver made of an optical filter, an ideal square-law photodetector, and an electrical filter was evaluated by using the time domain Karhunen-Loève expansion method. This approach was used to study the dependence of the bit error rate on waveguide and optical signal parameters. In particular, we have determined the domain in the system parameters space in which the signal degradation remains below a certain threshold used in practical settings to assess the fidelity of detected signals. It should be noted that the method introduced here can be easily extended to other silicon based components of on-chip and chip-tochip optical networks, including modulators, amplifiers, optical switches, and frequency converters. Equally important, our formalism can be applied to physical settings in which additional optical effects can become important. For example, our approach could readily incorporate nonlinear effects such as four-wave mixing and stimulated Raman scattering, which can become large enough to affect the bit error rate in properly designed waveguides or for shorter optical pulses. 


\section{REFERENCES}

[1] F. Benner, M. Ignatowski, J. A. Kash, D. M. Kuchta, and M. B. Ritter, "Exploitation of optical interconnects in future server architectures," IBM J. Res. Dev., vol. 49, no. 4, pp. 755-775, Jul.-Sep. 2005.

[2] K. Shacham, K. Bergman, and L. P. Carloni, "Photonic networks-on-chip for future generations of chip multiprocessors," IEEE Trans. Comput., vol. 57, no. 9, pp. 1246-1260, Sep. 2008.

[3] J. A. Kash, A. F. Benner, F. E. Doany, D. M. Kuchta, B. G. Lee, P. K. Pepeljugoski, L. Schares, C. L. Schow, and M. Taubenblatt, "Optical Interconnects in Exascale Supercomputers," 23rd Annual Meeting of the IEEE Photonics Society, pp. 483-484, Nov. 2010.

[4] A. Benner, D. M. Kuchta, P. K. Pepeljugoski, R. A. Budd, G. Hougham, B. V. Fasano, K. Marston, H. Bagheri, E. J. Seminaro, H. Xu, D. Meadowcroft, M. H. Fields, L. McColloch,M. Robinson, F.W. Miller, R. Kaneshiro, R. Granger, D. Childers, and E. Childers, "Optics for HighPerformance Servers and Supercomputers," presented at OTuH1, Optical Fiber Communication Conf. and Expo. (OFC), Mar. 23, 2010.

[5] T. Barwicz, H. Byun, and F. Gan, C. W. Holzwarth, M. A. Popovic, P. T. Rakich, M. R. Watts, E. P. Ippen, F. X. Kartner, H. I. Smith, J. S. Orcutt, R. J. Ram, V. Stojanovic, O. O. Olubuyide, J. L. Hoyt, S. Spector, M. Geis, M. Grein, T. Lyszczarz, and J. U. Yoon, "Silicon photonics for compact, energy-efficient interconnects," J. Opt. Netw., vol. 6, no. 1, pp. 63-73, Jan. 2007.

[6] J. S. Orcutt, A. Khilo, C. W. Holzwarth, M. A. Popovic, H. Li, J Sun, T. Bonifield, R. Hollingsworth, F. X. Krtner, H. I. Smith, V. Stojanovic, R. J. Ram, "Nanophotonic integration in state-of-the-art CMOS foundries," Opt. Exp., vol. 19, no. 3, pp. 2335-2346, Jan. 2011

[7] A. V. Krishnamoorthy, X. Zheng, G. Li, J. Yao, T. Pinguet, A. Mekis, H. Thacker, I. Shubin, Y. Luo, K. Raj, and J. E. Cunningham, "Exploiting CMOS Manufacturing to Reduce Tuning Requirements for Resonant Optical Devices," IEEE Photon. J., vol. 3, no. 3, pp. 567-579, Jun. 2011.

[8] R. Claps, D. Dimitropoulos, V. Raghunathan, Y. Han, and B. Jalali, "Observation of stimulated Raman amplification in silicon waveguides," Opt. Exp., vol. 11, no. 7, pp. 1731-1739, Jul. 2003.

[9] R. Espinola, J. I. Dadap, R. M. Osgood, S. J. McNab, and Y. A. Vlasov, "Raman amplification in ultrasmall silicon-on-insulator wire waveguides," Opt. Exp., vol. 12, no. 16, pp. 3713-3718, Aug. 2004.

[10] G. Cocorullo, M. Iodice, I. Rendina, and P. M. Sarro, "Silicon Thermooptic Micromodulator with 700-kHz -3-dB Bandwidth," IEEE Photon. Technol. Lett., vol. 7, no. 4, pp. 363-365, Apr. 1995.

[11] A. Liu, R. Jones, L. Liao, D. Samara-Rubio, D. Rubin, O. Cohen, R. Nicolaescu, and M. Paniccia, "A high-speed silicon optical modulator based on a metal-oxide-semiconductor capacitor," Nature, vol. 427, no. 6975, pp. 615-618, Feb. 2004.

[12] Q. Xu, B. Shmidt, S. Pradhan, and M. Lipson, "Micrometre-scale silicon electro-optic modulator," Nature, vol. 435, no. 7040, pp. 325-327, May 2005.

[13] R. L. Espinola, M.-C. Tsai, J. T. Yardley, and R. M. Osgood Jr., "Fast and low-power thermooptic switch on thin silicon-on-insulator," IEEE Photon. Technol. Lett., vol. 15, no. 10, pp. 1366-1368, Oct. 2005.

[14] O. Boyraz, P. Koonath, V. Raghunathan, and B. Jalali, "All optical switching and continuum generation in silicon waveguides," Opt. Exp., vol. 12, no. 17, pp. 4094-4102, Aug. 2004.

[15] B. G. Lee, A. Biberman, P. Dong, M. Lipson, and K. Bergman, "Alloptical comb switch for multiwavelength message routing in silicon photonic networks," IEEE Photon. Technol. Lett., vol. 20, no. 10, pp. 767-769, May 2008.

[16] P. C. P. Chen, A. M. Pappu, and A. B. Apsel, "Monolithic integrated SiGe optical receiver and detector," in Proc. Conf. Lasers and ElectroOptics, Tech. Dig. (CD) (Optical Society of America), Baltimore, MD, 2007, pp. 1-2, Paper CTuZ4.

[17] S. Assefa, F. Xia, W. M. J. Green, C. L. Schow, A. V. Rylyakov, and Y. A. Vlasov, "CMOS-Integrated Optical Receivers for On-Chip Interconnects," IEEE J. Sel. Top. Quantum Electron., vol. 16, no. 5, pp. 1376-1385, Sep.-Oct. 2010.

[18] H. Fukuda, K. Yamada, T. Shoji, M. Takahashi, t. Tsuchizawa, T Watanabe, J. Takahashi, and S. Itabashi, "Four-wave mixing in silicon wire waveguides," Opt. Exp., vol. 13, no. 12, pp. 4629-4637, Aug. 2005.

[19] S. Zlatanovic, J. S. Park, S. Moro, J. M. C. Boggio, I. B. Divliansky, N. Alic, S. Mookherjea, and S. Radic, "Mid-infrared wavelength conversion in silicon waveguides using ultracompact telecom-band-derived pump source," Nature Photon., vol. 4, no. 8, pp. 561-564, Aug. 2010.

[20] R. Ho, K. W. Mai, and M. A. Horowitz, "The future of wires," Proc. IEEE, vol. 89, no. 4, pp. 490-504, Apr. 2001.
[21] K. K. Lee, D. R. Lim, H. C. Luan, A. Agarwal, J. Foresi, and L. C. Kimerling, "Effect of size and roughness on light transmission in a $\mathrm{Si} / \mathrm{SiO}_{2}$ waveguide: Experiments and model," Appl. Phys. Lett., vol. 77, no. 11 , pp. 1617-1619, Sep. 2010.

[22] B. G. Lee, X. Chen, A. Biberman, X. Liu, I-W. Hsieh, C. Chou, J. I. Dadap, F. Xia, W. M. J. Green, L. Sekaric, Y. A. Vlasov, R. M. Osgood, and K. Bergman, "Ultrahigh-Bandwidth Silicon Photonic Nanowire Waveguides for On-Chip Networks," IEEE Photon. Technol. Lett., vol. 20, no. 6, pp. 398-400, Mar. 2008.

[23] X. Chen, N. C. Panoiu, and R. M. Osgood, "Theory of Raman-mediated pulsed amplification in silicon-wire waveguides," IEEE J. Quantum Electron., vol. 42, no. 1, pp. 160-170, Jan.-Feb. 2006.

[24] A. Papoulis, Probability, Random Variables, and Stochastic Processes, 3rd ed. New York, NY: McGraw-Hill, 1991, p. 329.

[25] G. Bosco, A. Carena, V. Curri, R. Gaudino, P. Poggiolini, and S. Benedetto, "A novel analytical approach to the evaluation of the impact of fiber parametric gain on the bit error rate," IEEE Trans. Commun., vol. 49, no. 12, pp. 2154-2163, Dec. 2001.

[26] E. Forestieri and M. Secondini, "On the Error Probability Evaluation in Lightwave Systems With Optical Amplification," J. Lightwave Technol., vol. 27, no. 6, pp. 706-717, Mar. 2009.

[27] R. M. Osgood, N. C. Panoiu, J. I. Dadap, X. Liu, X. Chen, I-W. Hsieh, E. Dulkeith, W. M. J. Green, and Y. A. Vlassov, "Engineering nonlinearities in nanoscale optical systems: physics and applications in dispersionengineered silicon nanophotonic wires," Adv. Opt. Photon., vol. 1, no. 1, pp. 162-235, Jan. 2009.

[28] Q. Lin, O. J. Painter, and G. P. Agrawal, "Nonlinear optical phenomena in silicon waveguides: Modeling and applications," Opt. Exp., vol. 15, no. 25, pp. 16604-16644, Dec. 2007.

[29] J. I. Dadap, N. C. Panoiu, X. G. Chen, I. W. Hsieh, X. P. Liu, C. Y. Chou, E., Dulkeith, S. J., McNab, F. N. Xia, W. M. J. Green, L. Sekaric, Y. A. Vlasov, and R. M. Osgood, "Nonlinear-optical phase modification in dispersion-engineered Si photonic wires," Opt. Exp., vol. 16, no. 2, pp. 1280-1299, Jan. 2008.

[30] S. Lavdas, J. B. Driscoll, R. R. Grote, R. M. Osgood, and N. C. Panoiu, "Pulse compression in adiabatically tapered silicon photonic wires," Opt. Exp., vol. 22, no. 6, pp. 6296-6312, Mar. 2014.

[31] K. Kikuchi, "Enhancement of optical-amplifier noise by nonlinear refractive index and group-velocity dispersion of optical fibers," IEEE Photon. Technol. Lett., vol. 5, no. 2, pp. 221-223, Feb. 1993.

[32] C. W. Helstrom, Statistical Theory of Signal Detection. New York: Pergamon, 1968, ch. IV.

[33] A. M. Mathai and S. B. Provost, Quadratic Forms in Random Variables. New York, NY: Marcel Dekker, 1992, ch. III.

[34] C. W. Helstrom, "Distribution of the Filtered Output of a Quadratic Rectifier Computed by Numberical Contour Integration," IEEE Trans. Inf. Theory, vol. IT-32, no. 4, pp. 450-463, Jul. 1986.

[35] N. S. Bergano, F. W. Kerfoot, and C. R. Davidson, "Margin Measurements in Optical Amplifier Systems," IEEE Photon. Technol. Lett., vol. 5, no. 3, pp. 304-308, Mar. 1993.

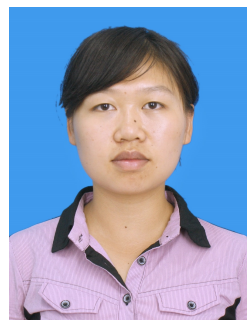

Jie You was born in Hunan province, China, in 1991. She received the B.Sc. degree in optical information science and technology from National University of Defense Technology, Changsha, China, in 2013. She is currently working toward the Ph.D. degree in Electrical Engineering at University College London, London, UK. Her current research interests are focused on silicon optical interconnects for chipscale communications and optical pulse propagation in silicon photonic wires.

Nicolae C. Panoiu received the B.Sc. and M.S. degrees in physics from the University of Bucharest, Bucharest, Romania, in 1990 and 1992, respectively, and the Ph.D. degree from New York University, New York, in 2001. After graduating from NYU, he was a Postdoctoral Fellow in the Department of Applied Physics and Applied Mathematics, Columbia University, New York. $\mathrm{He}$ is currently Reader in Nanophotonics in the Department of Electronic and Electrical Engineering, University College London. His research interests include silicon photonics, optical properties of photonic nanostructures and metamaterials, and computational modeling of electromagnetic structures. Dr. Panoiu is a member of the OSA. 